\title{
Gestão de cadeias de suprimentos humanitárias: perspectivas teóricas e direcionamentos futuros
}

\section{Humanitarian Supply Chain Management: Theoretical Perspectives and Future Directions}

\author{
GLESSIA SILVA*
}

\section{RESUMO}

Este artigo tem como objetivo discutir como se encontra a literatura em gestão de cadeias de suprimentos humanitárias e os direcionamentos futuros desse campo. Para tanto, foi realizado um ensaio teórico sobre a temática. A literatura em gestão de cadeias de suprimentos humanitárias está pautada em estudos voltados ao relacionamento nas cadeias, papel dos stakeholders, gerenciamento da cadeia e logística humanitária. Como direcionamentos futuros, observa-se uma tendência na compreensão do conceito de desastre como particular a cada localidade, o papel da mídia na mobilização de recursos, maior ênfase no papel dos stakeholders na efetividade dessas cadeias e a influência dessas cadeias na gestão de cadeias de suprimentos comerciais. Como contribuições são sugeridas oportunidades de pesquisa no campo

Universidade Federal de Sergipe - UFS. Pós-Doutorado em Administração de Empresas pela Fundação Getulio Vargas, na Escola de Administração de Empresas de São Paulo FGV/EAESP (2019-Atual). Doutora em Administração de Empresas pela Fundação Getulio Vargas, na Escola de Administração de Empresas de São Paulo - FGV/EAESP (2018). Mestra em Administração pela Universidade Federal de Sergipe - UFS (2013). Bacharela em Administração pela Universidade Federal de Sergipe - UFS (2011). Tem interesse de pesquisa em: inovação, pequena empresa e desenvolvimento. Lecionou na Universidade Federal de Sergipe como Professora Substituta do Departamento de Administração (2013). Lecionou na Universidade Federal de Alagoas, Campos do Sertão, como Professora Assistente do Eixo Tecnologia (2013-2018). Lecionou no Mestrado Profissional em Administração Pública em Rede Nacional - PROFIAP (2019). Atualmente é Professora Adjunta da Universidade Federal de Sergipe, no Departamento de Administração (2018-Atual) e no Mestrado Acadêmico em Administração - PROPADM (2019-Atual). Foi Assistente de Pesquisa do Fórum de Inovação da FGV/EAESP (2015-2017). E-mail: glessiasilva@hotmail.com 
de gestão de cadeias de suprimentos humanitárias. Espera-se que este estudo sirva de ponto de partida para outros estudos e estimule o pensamento crítico acerca desse emergente campo de estudo.

Palavras-chave: Cadeias Humanitárias. Operações. Gestão de Cadeias de Suprimentos Humanitárias.

\section{Abstract}

This article aims to discuss how the literature on the management of humanitarian supply chains and the future directions of this field are found. For that, a theoretical essay on the subject was carried out. The literature on the management of humanitarian supply chains is based on studies related to chain relationships, stakeholder roles, chain management and humanitarian logistics. As future directions, there is a tendency to understand the disaster concept as particular to each locality, the media's role in mobilizing resources, and a greater emphasis on the role of stakeholders in the effectiveness of these chains, as well as the influence of these chains in the management of commercial supply chain. As a contribution we suggest research opportunities in the field of humanitarian supply chain management. We hope that this study will serve as a starting point for further studies and stimulate critical thinking about this emerging field of study. Keywords: Humanitarian Chains. Operations. Humanitarian Supply Chain Management.

\section{INTRODUÇÃO}

A gestão de cadeias de suprimentos humanitárias inclui as atividades de preparação, planejamento, aquisição, transporte, armazenamento, rastreamento e desembaraço aduaneiro, desenvolvidas a fim de minimizar o sofrimento humano e oferecer tratamento imediato para aqueles com lesões e doenças (ABIDI; LEEUW; KLUMPP, 2014; ANTAI; MUTSHINDA; OWUSU, 2015; BLECKEN et al., 2009; CHANDRAPRAKAIKUL, 2010; COSTA; CAMPOS; BANDEIRA, 2012; ERTEM; BUYURGAN; ROSSETTI, 2010; YU et al., 2015). A gestão dessas cadeias ganhou destaque em virtude do aumento no número de desastres (BEN-TALA et al., 
2011; CHANDRAPRAKAIKUL, 2010; KOVÁCS; TATHAM, 2009; KOVÁCS; SPENS, 2011; CHANDES; PACHÉ, 2010; OLORUNTOBA, 2010; VAN DER LAAN; BRITO; VERGUNST, 2009). Nos últimos 14 anos houve em média 416 desastres naturais em todo o mundo e, desde o século passado, os desastres foram responsáveis por mais de 30 milhões de mortes (ANTAI; MUTSHINDA; OWUSU, 2015).

Dentre esses desastres, podem-se destacar globalmente o terremoto e tsunami do Oceano Índico (2004), o Furacão Katrina (2005), o terremoto do Haiti (2010), o acidente nuclear de Fukushima no Japão (2011), a Guerra da Síria (2011-atual), e o surto de Ebola na África (2014-2015); e no Brasil enchentes no Estado de São Paulo e demais Estados (atual), deslizamentos de terra no Rio de Janeiro (2011), Crise Hídrica no Estado de São Paulo (2014-2016), rompimento da barragem de Mariana em Minas Gerais (2015), rompimento da barragem de Brumadinho em Minas Gerias (2019) e surto de Zika no Nordeste Brasileiro (2015-2016).

A irregularidade das catástrofes e seu impacto sobre a vida das pessoas têm dificultado o planejamento, o desenvolvimento de pesquisas e a atuação de equipes de resgates, fazendo com que essas cadeias sejam mais pressionadas (ANTAI; MUTSHINDA; OWUSU, 2015). As graves perdas humanas e os sérios prejuízos econômicos e ambientais têm chamado a atenção para uma gestão mais eficiente (BEN-TALA et al., 2011; RENNEMO et al., 2014) e têm obrigado essas cadeias a se desenvolverem (CHANDES; PACHÉ, 2010). O mundo demonstra estar em crise (PAZIRANDEH, 2011) e o próximo desastre certamente está próximo de acontecer, seja na forma de epidemias, catástrofes naturais ou provocadas pelo homem, o que reforça ainda mais a importância de uma gestão eficiente (OVERSTREET et al., 2011).

Entretanto, a gestão de cadeias de suprimentos humanitárias envolve desafios. Se por um lado elas enfrentam questões semelhantes às cadeias comerciais, por outro a antecipação, proatividade e capacidade de resposta representam números de vidas e cada desastre possui características próprias, de forma que o sucesso na resposta a um desastre não garante o sucesso em outros (CHANDES; PACHÉ, 2010). O contexto único das cadeias de suprimentos humanitárias aumenta sua complexidade e dificulta um enquadramento de ações efetivas 
para todo tipo de cadeia (MCLACHLIN; LARSON, 2011), de forma que um gerenciamento adequado pode não apenas reduzir custos e tempo, mas salvar vidas e reduzir o sofrimento (DAY et al., 2012).

Dada essas considerações, este artigo tem como objetivo discutir como se encontra a literatura em gestão de cadeias de suprimentos humanitárias e os direcionamentos futuros desse campo. Foi realizado um ensaio teórico sobre a temática (MENEGHETTI, 2011) e foram considerados apenas trabalhos que usaram o termo "gestão de cadeias de suprimentos humanitárias", na língua portuguesa e inglesa, no título, resumo, ou corpo do trabalho. Isso porque o campo que trata da temática é amplo e o foco do artigo reside em compreender o enquadramento do aspecto gerencial das cadeias de suprimentos humanitárias. Se por um lado essa delimitação exclui uma quantidade considerável de trabalhos com diferentes enfoques, por outro ela permite atender especificamente os objetivos desta pesquisa. A autoidentificação do termo nos textos pesquisados revela a forma como os pesquisadores compreendem o gerenciamento dessas cadeias e pode revelar importantes insights de pesquisa.

Espera-se que os insights gerados contribuam para o desenvolvimento do campo de estudo que trata da gestão de cadeias de suprimentos humanitárias e possibilitem oportunidades de pesquisa.

\section{GESTÃO DE CADEIAS DE SUPRIMENTOS HUMANITÁRIAS}

A gestão de cadeias de suprimentos humanitárias inclui uma ampla variedade de atividades, que diferem das atividades das cadeias comerciais em âmbito temporal e espacial, objetivos, público-alvo, circunstâncias em que as operações são desenvolvidas e bens e serviços entregues (BALCIK; BEAMON, 2008; BALCIK et al., 2010; BLECKEN et al., 2009; RENNEMO et al., 2014). Essas cadeias também se diferenciam por serem múltiplas, dinâmicas, globais e temporárias, além de estarem em constante processo de abertura e fechamento (KOPCZAK; JOHNSON, 2007).

As cadeias de suprimentos humanitárias são afetadas por fatores específicos, associados ao tipo de desastre que estão operando, como: número e diversidade de atores, expectativa dos doadores e estrutura de investimento, competição para financiamento e efeito da mídia, imprevisibilidade, escassez de recursos e excesso de oferta, 
e custo de coordenação (BALCIK; BEAMON, 2008; BALCIK et al., 2010). Como desastre, tem-se qualquer tipo de ocorrência, causada pelo homem ou pela natureza, que provoque dano ou sofrimento humano, como catástrofes súbitas, enchentes, erupções vulcânicas, eventos globais e locais, problemas com alimentação, moradia e saúde (ADIVAR et al., 2010). Essa definição de desastre será a utilizada neste artigo.

A gestão de desastres inclui as fases de mitigação - esforços a longo prazo para evitar o desastre -; preparação - ações anteriores à ocorrência do desastre -; resposta - ações posteriores ao desastre; e recuperação - ações voltadas à restauração das pessoas e localidades afetadas (ADIVAR et al., 2010; AHMADI; SEIFI; TOOTOONI, 2015; COSTA et al., 2012; KOPCZAK; JOHNSON, 2007; KOVÁCS; SPENS, 2009; KOVÁCS; TATHAM, 2009). Toda sua gestão deve vir acompanhada de campanhas de sensibilização e educação, desenvolvimento de alertas específicos precoces, planos antes e pós-desastre (OLORUNTOBA, 2010), e a fase de pós-desastre deve envolver o governo como unidade de resposta, a atuação de unidades locais, um planejamento tático bem definido, execução de ERC - movimento global, "no qual empresas industriais e comerciais, juntamente com os demais integrantes da cadeia de abastecimento trabalham em conjunto na busca de padrões comuns e processos eficientes que permitam minimizar os custos e otimizar a produtividade em suas relações" - e planejamento estratégico (OLORUNTOBA, 2010).

Após um desastre, as organizações têm 24 horas para fazer o reconhecimento do mesmo e até 72 horas para liberar os recursos necessários ao socorro (ERTEM; BUYURGAN; ROSSETTI, 2010; WASSENHOVE, 2006). Nessas situações, a adequada gestão garante que os recursos necessários sejam destinados, além de reestabelecer o bem-estar das vítimas e garantir a reconstrução (ADIVAR et al., 2010). Para que isso ocorra deve haver planejamento e articulação entre diversos atores - governo, agências internacionais, ONGs internacionais, ONGs nacionais, agentes locais e comunitários, ajuda de países, sociedade civil, empresas (ERTEM; BUYURGAN; ROSSETTI, 2010; KOPCZAK; JOHNSON, 2007; WASSENHOVE, 2006), já que a fonte de receita para a cadeia de suprimentos humanitárias é o financiamento do governo e doações de pessoas da sociedade civil e 
empresarial (CHANDRAPRAKAIKUL, 2010). Em decorrência disso, em cadeias humanitárias há uma forte dependência entre mecanismos de controle e confiança, pois a confiança entre os parceiros é o que faz com que estas cadeias funcionem (KOPCZAK; JOHNSON, 2007; STEPHENSON, 2005; STEPHENSON; SCHNITZER, 2006).

Um dos principais desafios na gestão de cadeias de suprimentos humanitárias é a logística, que deve ser reconhecida e entendida como elemento intrínseco das operações de ajuda (WASSENHOVE, 2006), e corresponde a $80 \%$ dos custos totais em recuperação de desastres (IAKOVOU et al., 2014). Como atividades da logística humanitária estão: planejamento de distribuição, sistema de informação e comunicação, fornecimento e gestão de fornecedores, coordenação e integração da cadeia, avaliação de desempenho e opções de transporte (BALCIK; BEAMON, 2008; BALCIK et al., 2010; CHANDRAPRAKAIKUL, 2010). E entre os desafios: falta de padrões e indicadores, formação inadequada, falta de colaboração, baixo reconhecimento da logística e infraestrutura inadequada (KOPCZAK; JOHNSON, 2007; KOVÁCS; SPENS, 2009).

A gestão das cadeias de suprimentos humanitárias permite a redução de falhas (ANTAI; MUTSHINDA; OWUSU, 2015), que são deficiências na prestação da ajuda. Essas falhas podem não ser facilmente identificadas, em virtude da magnitude, frequência, tempo, duração, área e velocidade do desastre (ANTAI; MUTSHINDA; OWUSU, 2015). Nesse sentido, deve-se compreender quais são os drivers e barreiras que afetam o desempenho dessas cadeias, bem como os aspectos culturais, organizacionais, tecnológicos, individuais e estratégicos da cadeia humanitária em questão e da localidade em que ocorreu o desastre (KABRA; RAMESH, 2015).

\section{0 QUE NOS DIZ A LITERATURA DE GESTÃo DE CADEIAS DE SUPRIMENTOS HUMANITÁRIAS?}

Os estudos sobre cadeias de suprimentos humanitárias se desenvolveram intensamente na última década (CHANDRAPRAKAIKUL, 2010; KOVÁCS; SPENS, 2011; CHANDES; PACHÉ, 2010; MCLACHLIN; LARSON, 2011; OLORUNTOBA, 2010; VAN DER LAAN; BRITO; VERGUNST, 2009) e trouxeram com eles um campo que emergiu da prática, mas que já dá sinais de enquadra- 
mento teórico. Observa-se, assim, uma tendência de migração do foco em logística humanitária para uma compreensão mais voltada à gestão de cadeias de suprimentos humanitárias.

A coordenação de atividades logísticas foi pauta constante em alguns estudos, que buscaram compreender melhor os mecanismos necessários para uma boa logística humanitária (BEN-TALA et al., 2011; CHANDES; PACHÉ, 2009; IAKOVOU et al., 2014; KOVÁCS; SPENS, 2009; MARTINEZ; STAPLETON; VAN WASSENHOVE, 2011; MAYS; GUGERTY; RACADIO, 2012; WASSENHOVE, 2006). Isso ocorre porque na gestão de cadeias de suprimentos humanitárias a atividade logística se apresenta como uma atividade chave nas etapas de desastre e é difícil sua coordenação no momento do desastre, dado o alto nível de incerteza e a dificuldade de alocar diferentes recursos na operação (CHANDES; PACHÉ, 2009; IAKOVOU et al., 2014), sendo crucial a formação de uma "estratégia coletiva" que promova essa mobilização (CHANDES; PACHÉ, 2009).

O desempenho em cadeias de suprimentos humanitárias também foi outro ponto de investigação, com estudos voltados a modelos para melhorar o desempenho da cadeia em casos de desastre (BEAMON; BALCIK, 2008; BLECKEN et al., 2009; VAN DER LAAN; BRITO; VERGUNST, 2009). Medir o desempenho em cadeias de suprimentos humanitárias ainda se mostra desafiador, já que por um lado a gestão de desempenho deve estar intimamente ligada à estratégia da cadeia e por outro os sistemas de medição de desempenho não são amplamente implementados ao longo da cadeia e tão pouco fazem parte de sua estratégia (ABIDI; LEEUW; KLUMPP, 2014). Além disso, apesar das cadeias humanitárias serem geridas com base em princípios extraídos da gestão de cadeias de suprimentos comerciais, suas peculiaridades exigem métricas de desempenho diferenciadas, o que aumenta ainda mais a complexidade na medição do desempenho (BEAMON; BALCIK, 2008).

Outra perspectiva enfatiza que muitos doadores se mostram desapontados com as respostas negativas para catástrofes e discute como a confiança e a parceria pode ser recuperada em cadeias humanitárias, havendo a necessidade de estruturas organizacionais com cadeias de comando claras e clara demarcação de tomada de decisão, autoridade e responsabilidade (KOPCZAK; JOHNSON, 
2007; STEPHENSON; SCHNITZER, 2006). Esse estudo, em conjunto com os estudos desenvolvidos por Herlinb e Pazirandeh (2012), Pazirandeh (2011) e Pazirandeh e Herlin (2014), chama a atenção para a importância do relacionamento na cadeia e destaca o papel dos stakeholders em sua gestão.

O processo de tomada de decisão e as alternativas de decisão a serem seguidas em caso de catástrofe também é uma possibilidade de estudo, já que boa parte das decisões são tomadas com base na experiência e os efeitos dessas decisões refletem perdas humanas e não apenas monetárias, como nas cadeias comerciais, o que chama a atenção para a criação de modelos de tomada de decisão adequados ao contexto dessas cadeias (BESIOU; STAPLETON; VAN WASSENHOVE, 2011).

Observaram-se também estudos voltados à busca por competências ou fatores chave de sucesso na gestão de cadeias de suprimentos humanitárias (BESIOU; STAPLETON; VAN WASSENHOVE, 2011; OLORUNTOBA, 2010; PELLEGRIN-ROMEGGIO; VEGA, 2014). Oloruntoba (2010) argumenta que ainda existe pouca literatura sobre os elementos de sucesso em casos de boa resposta e que estes elementos poderiam servir para traçar ações em países em desenvolvimento, que concentram a maior parte dos desastres, sendo eles: uso efetivo de sistemas de informação e de comunicação; efetiva gestão da cadeia de suprimentos, que deve ser participativa, colaborativa e envolver todos os interessados; e boa gestão de stakeholders. Já Pellegrin-Romeggio e Veja (2014) propõem uma competência que permita à cadeia combinar dinamicamente - montar/desmontar e ativar/desativar - os recursos conforme necessário, e introduzem o conceito de "conjunto dinâmico", que integra esta nova competência em capacidades base para integrar redes temporárias.

Uma das características das cadeias humanitárias é sua mobilização em um curto período de tempo e a necessidade de coordenação durante um longo período de tempo para garantir que a mobilização ocorra sempre que necessário, o que exige agilidade (COZZOLINO; ROSSI; CONFORTI, 2012; OLORUNTOBA; GRAY, 2006). A adoção de princípios leans e ágeis na cadeia de suprimentos humanitárias tem sido, portanto, discutida como forma de poupar tempo na resposta a desastres e salvar vidas (COZZOLINO; ROSSI; 
CONFORTI, 2012; OLORUNTOBA; GRAY, 2006) e traz a tona a necessidade de estudos empíricos que possam testar o funcionamento de um "modelo de cadeia de suprimento humanitária" (OLORUNTOBA; GRAY, 2006).

Antai, Mutshinda e Owusu (2015), tratam das falhas de cadeias de suprimentos humanitárias e da resposta a desastres naturais, com base no princípio dos 3R: hora certa, lugar certo e materiais certos. Esses três princípios seriam a base da cadeia e direcionariam os esforços e decisões em caso de desastres. Por fim, Leiras et al. (2014) apontam haver uma lacuna na literatura que trata de recuperação. A recuperação é a última fase do desastre e reflete as ações necessárias à "recuperação" do que foi afetado (ADIVAR et al., 2010; AHMADI; SEIFI; TOOTOONI, 2015; COSTA; CAMPOS; BANDEIRA, 2012; KOPCZAK; JOHNSON, 2007; KOVÁCS; SPENS, 2009; KOVÁCS; TATHAM, 2009). Como a literatura que trata de gestão de cadeias de suprimentos humanitárias ainda é recente e emergiu da prática, é esperado que a literatura reflita as fases anteriores à fase de recuperação, já que os países em desenvolvimento concentram a maior parte dos desastres (OLORUNTOBA, 2010) e, portanto, servem de campo para a maior parte dos estudos, e ainda não dispõem de mecanismos tão eficientes para lidar com as etapas anteriores.

\section{Direcionamentos FUturos}

A gestão de cadeias de suprimentos humanitárias envolve múltiplos desafios e, portanto, múltiplas oportunidades de pesquisa. Espera-se que os insights gerados a seguir sirvam de ponto de partida para outros estudos e estimulem o pensamento crítico acerca desse emergente campo de estudo.

\subsection{Desastre?}

O conceito de desastre demonstra ser específico de cada localidade ou país, bem como compreende diferentes tipos de enquadramentos na literatura. O conceito de desastre compreende qualquer tipo de ocorrência, causada pelo homem ou pela natureza, que provoque dano ou sofrimento humano (ADIVAR et al., 2010; ANTAI; MUTSHINDA; OWUSU, 2015; CHANDES; PACHÉ, 2010; COSTA; CAMPOS; BANDEIRA, 2012; KOVÁCS; SPENS, 2009; 
ROTTKEMPER; FISCHER; BLECKEN, 2012; VAN WASSENHOVE, 2006). Esses desastres podem ser: catástrofes súbitas, enchentes, erupções vulcânicas, eventos globais e locais, problemas com alimentação, moradia e saúde (ADIVAR et al., 2010); desastres de início súbito (terremoto e deslizamento, incêndio florestal, industrial e poluição da água) e desastres de início lento (erosão costeira, afluxo de refugiados e repatriados) (KOVÁCS; SPENS, 2009).

Embora seja grande o número de pessoas afetadas por desastres, esse é ainda maior se considerado o número de desastres não noticiados pela mídia por não repercutirem ou não serem considerados "manchete", as chamadas "crises humanitárias esquecidas", a exemplo de civis vítimas de bombardeio, vítimas da falta de investimento contra o HIV e outras doenças em algumas regiões, assim como miséria e fome (ROTTKEMPER; FISCHER; BLECKEN, 2012). Em torno de $90 \%$ das pessoas vítimas de desastres são vítimas das "crises humanitárias esquecidas" e vivem em países em desenvolvimento (ROTTKEMPER; FISCHER; BLECKEN, 2012). Apesar disso, a maior parte dos estudos que trata de cadeias de suprimentos humanitárias está voltada a desastres naturais.

Isso significa que o conceito de desastre está difuso e que boa parte dos desastres está de fato sendo esquecida e, indo além, que muitos países são considerados zonas livres de desastres quando na verdade guardam as "crises humanitárias esquecidas".

\subsection{E a mídia?}

A mídia exerce um forte papel na repercussão do desastre e mobilização de recursos, dado o seu poder de mexer com o apelo social (BALCIK et al., 2010; HERLINB; PAZIRANDEH, 2012; ROTTKEMPER; FISCHER; BLECKEN, 2012; WASSENHOVE, 2006). Segundo estudo realizado por Maghsoudi e Pazirandeh (2016) em 101 organizações humanitárias do sudeste asiático, “a visibilidade tem um impacto significativo sobre a partilha de recursos e o desempenho das organizações, especialmente em termos da vontade de partilhar recursos, recursos utilizados e flexibilidade das organizações". Isso vem sendo feito principalmente em desastres naturais e em situações que envolvam um desastre raro (HERLINB; PAZIRANDEH, 2012). 
Entretanto, nas "crises humanitárias esquecidas", onde os recursos são ainda mais escassos, havendo, portanto, a necessidade de mecanismos que ajudem a angariar mais doações e investimentos, observa-se pouca atuação da mídia. Tal situação se mostra contraditória, já que a atenção dada pela mídia, ao que parece ser um "desastre de interesse público", por um lado auxilia no aumento de doações para alguns desastres, mas por outro traz uma influência negativa para as "crises humanitárias esquecidas", que parecem não existir aos olhos de seus mantenedores.

\subsection{STAKeHolders: A BASE dA CADEIA}

A necessidade de resposta rápida para os desastres tem exigido maior articulação entre diversos agentes - governo, agências internacionais, ONGs internacionais, ONGs nacionais, agentes locais e comunitários, ajuda entre países, fornecedores, empresas - e colocado cada vez mais em discussão o seu papel em casos de desastre (AHMADI; SEIFI; TOOTOONI, 2015; BALCIK et al., 2010; SCHULZ; BLECKEN, 2010; VEJA; ROUSSAT, 2015). O envolvimento do setor privado também se faz cada vez mais presente, já que essas organizações viram suas operações fragilizadas em virtude de alguns dos principais desastres naturais da história (DAY et al., 2012; VAN WASSENHOVE, 2006).

A coordenação de cadeias de suprimentos humanitárias envolve múltiplos atores com interesses diferentes e capacidades e expertises específicas (BALCIK et al., 2010; MAON; LINDGREEN; VANHAMME, 2009; SCHULZ; BLECKEN, 2010). A maior dificuldade é, portanto, gerir os diferentes interesses (CAMPOS, 2012; OLORUNTOBA, 2010). A falta de coordenação nas cadeias de suprimentos humanitárias dificulta as operações de salvamento e socorro, o que aumenta ainda mais a necessidade de maior articulação entre o governo e o empresariado, em prol de ações mais efetivas (KABRA; RAMESH, 2015; PAZIRANDEH, 2011).

Um agente cada vez mais relevante são as organizações sem fins lucrativos, que, embora estejam desempenhando um papel cada vez mais importante nas cadeias humanitárias, tanto em impactos sociais, quanto em mobilização de empresas e outras entidades, 
são subpesquisadas na literatura que trata de cadeias humanitárias (HERLINB; PAZIRANDEH, 2012).

\subsection{Quais teorias servem de suporte?}

Um desafio no campo da gestão de cadeias de suprimentos humanitárias é que os conceitos ainda estão pouco amadurecidos e insuficientes (OVERSTREET et al., 2011) e, por serem complexas, há a necessidade de pensar nas cadeias de suprimentos humanitárias do ponto de vista da gestão de cadeias de suprimentos comerciais (YU et al., 2015).

Em relação às tentativas de compreensão dessas cadeias sob uma lente teórica, tem-se o uso da teoria da dependência de recursos para compreender a distribuição de poder ao longo da cadeia (HERLINB; PAZIRANDEH, 2012). Ela constitui uma boa lente por permitir entender peculiaridades desse tipo de cadeia, que podem ser analisadas com base nos três fatores críticos na dependência de recursos de Pfeffer e Salancik (1978): importância do recurso, que no caso dessas cadeias significa vidas; prudência na alocação e uso dos recursos, onde recursos escassos devem atender o maior número possível de vítimas em um momento pouco previsível; e as poucas alternativas existentes, onde se observa a necessidade de engajamento de múltiplos stakeholders.

A teoria sistêmica também foi aplicada, sendo utilizada para compreender a cadeia humanitária como um sistema aberto, complexo e adaptativo, cuja interação entre estrutura e processos pode explicar a qualidade da resposta (SEYBOLT, 2009); e o uso da teoria da contingência para analisar o aspecto social destas cadeias e como lidam com o ambiente de incerteza presente em suas atividades (HAAVISTO; KOVÁCS, 2014). Sobre o último, os autores empregam o conceito de sustentabilidade em cadeias humanitárias como a capacidade de continuar depois que o financiamento dos doadores for retirado, o que impacta diretamente a continuidade das ações humanitárias em longo prazo.

\subsection{Da prática à teoria ou da teoria à prática?}

Embora o campo de gestão de cadeias de suprimentos humanitárias tenha nascido da prática, há a necessidade de integrar 
soluções práticas e teóricas para o contexto atual dessas cadeias (COSTA; CAMPOS; BANDEIRA, 2012). Como discutido nos tópicos anteriores, ainda existem múltiplas oportunidades de pesquisa que podem contribuir para o amadurecimento do campo e para a consolidação de aspectos conceituais que direcionem a prática nessas cadeias. Além disso, apesar de tomarem emprestadas diversas aplicações das cadeias de suprimentos comerciais, as cadeias de suprimentos humanitárias podem promover importantes lições às cadeias comercias, já que operam em condições de extrema incerteza e pressão (COSTA; CAMPOS; BANDEIRA, 2012). Cabe, portanto, pensar nas lições que as cadeias de suprimentos humanitárias têm a oferecer às organizações.

\section{Considerações Finais}

Este artigo teve como objetivo discutir como se encontra a literatura em gestão de cadeias de suprimentos humanitárias e os direcionamentos futuros desse campo. Trata-se de uma temática que tem ganhado destaque nos últimos anos em virtude dos impactos sociais e econômicos causados pelos desastres. As cadeias de suprimentos humanitárias surgem com a necessidade de prestar socorro e responder de forma rápida em momentos de desastres para minimizar os possíveis impactos ou danos, o que exige a articulação de diversos stakeholders.

É preciso ressaltar que a literatura tem evidenciado de forma crítica o papel da mídia, que trata dos desastres humanitários dando maior destaque para aqueles que repercutem mundialmente, ao passo que ignora as "crises humanitárias esquecidas" que, em geral, ocorrem nos países pobres. A Fome na África não é noticiada pela mídia com o mesmo afinco que a Crise de Imigração que atinge a Europa, por exemplo, apesar do maior número de pessoas afetadas por essas "crises humanitárias esquecidas". É preciso, portanto, revisar o próprio conceito de "desastre" e o entendimento sobre os papéis dos diversos stakeholders. Com isso, espera-se ampliar o escopo de discussão sobre o gerenciamento das cadeias de suprimentos humanitárias e qual seria de fato seu escopo de atuação.

Observa-se também a tendência da literatura sobre "gestão de cadeias de suprimentos humanitárias" utilizar o termo dessa manei- 
ra, com a definição constitutiva mais ampla, pois denota maior nível de coordenação e articulação necessário à efetividade dessas cadeias; bem como a possibilidade de aplicação dos preceitos adotados nas cadeias de suprimentos humanitárias nas cadeias de suprimentos comerciais. Percebe-se, ainda, uma tendência de "humanização" na área de operações, tradicionalmente mais pragmática e técnica, mas que no contexto das cadeiras humanitárias passa a relacionar ao seu escopo elementos mais humanos e direcionados a questões sociais.

Esses insights trazem implicações práticas e acadêmicas para o campo de gestão de cadeias de suprimentos humanitárias. Como implicações práticas, nota-se uma tendência de interdisciplinaridade nesse campo de estudo e atuação, pois o profissional de cadeias de suprimentos humanitárias, além dos conhecimentos exigidos aos profissionais de cadeias de suprimentos comerciais, necessita ter algum nível de conhecimento em antropologia ou sociologia, por exemplo, dada a necessidade de atuar em contextos culturais diversos e adversos. Além disso, o artigo pode servir de reflexão para os stakeholders que compõem as cadeias de suprimentos humanitárias. Como implicações acadêmicas, o artigo traz oportunidades de pesquisa e contribui para o fortalecimento da literatura que trata de gestão de cadeias de suprimentos humanitárias.

Como sugestões para pesquisas futuras, destacam-se as "crises humanitárias esquecidas" como um objeto de estudo em potencial, e os aspectos de interdisciplinaridade como forma de incorporar os conhecimentos de outros campos para enriquecer o campo de operações.

\section{REFERÊNCIAS}

ABIDI, H.; DE LEEUW, S.; KLUMPP, M. Humanitarian supply chain performance management: a systematic literature review. Supply Chain Management: An International Journal, vol. 19, n. 5/6, pp. 592-608, 2014.

ADIVAR, B.; ATAN, T.; SEVIL OFLAÇ, B.; ÖRTEN, T. Improving social welfare chain using optimal planning model. Supply Chain Management: An International Journal, vol. 15, n. 4, pp. 290-305, 2010.

AHMADI, M.; SEIFI, A.; TOOTOONI, B. A humanitarian logistics model for disaster relief operation considering network failure and standard relief time: A case study on San Francisco district. Transportation Research Part E: Logistics and Transportation Review, vol. 75, pp. 145-163, 2015. 
AKHTAR, P.; MARR, N. E.; GARNEVSKA, E. V. Coordination in humanitarian relief chains: chain coordinators. Journal of humanitarian logistics and supply chain management, vol. 2, n. 1, pp. 85-103, 2012.

ANTAI, I.; MUTSHINDA, C.; OWUSU, R. A 3-R principle for characterizing failure in relief supply chains' response to natural disasters. Journal of Humanitarian Logistics and Supply Chain Management, vol. 5, n. 2, pp. 234-252, 2015.

BALCIK, B.; BEAMON, B. M. Facility location in humanitarian relief. International Journal of Logistics, vol. 11, n. 2, pp. 101-121, 2008.

BALCIK, B.; BEAMON, B. M.; KREJCI, C. C.; MURAMATSU, K. M.; RAMIREZ, M. Coordination in humanitarian relief chains: Practices, challenges and opportunities. International Journal of Production Economics, vol. 126, n. 1, pp. 22-34, 2010.

BEAMON, B. M.; BALCIK, B. Performance measurement in humanitarian relief chains. International Journal of Public Sector Management, vol. 21, n. 1, pp. 4-25, 2008.

BEN-TAL, A.; DO CHUNG, B.; MANDALA, S. R.; YAO, T. Robust optimization for emergency logistics planning: Risk mitigation in humanitarian relief supply chains. Transportation Research Part B: Methodological, vol. 45, n. 8, pp. 1177-1189, 2011.

BESIOU, M.; STAPLETON, O.; VAN WASSENHOVE, L. N. System dynamics for humanitarian operations. Journal of Humanitarian Logistics and Supply Chain Management, vol. 1, n. 1, pp. 78-103, 2011.

BLECKEN, A.; HELLINGRATH, B.; DANGELMAIER, W.; SCHULZ, S. F. A humanitarian supply chain process reference model. International Journal of Services Technology and Management, vol. 12, n. 4, pp. 391-413, 2009.

CHANDES, J.; PACHÉ, G. To Ponder on the Collective Actions in the Context of Humanitarian Logistics: Lessons from the Earthquake in Pisco. Journal of Economics, Finance \& Administrative Science, vol. 14, n. 28, 2009.

CHANDES, J.; PACHÉ, G. Investigating humanitarian logistics issues: from operations management to strategic action. Journal of Manufacturing Technology Management, vol. 21, n. 3, pp. 320-340, 2010.

CHANDRAPRAKAIKUL, W. Humanitarian Supply Chain Management: Literature Review and Future Research. In The 2nd International Conference on Logistics and Transport, Queenstown, New Zealand, 2010.

COSTA, S. R. A.; CAMPOS, V. B. G.; DE MELLO BANDEIRA, R. A. Supply chains in humanitarian operations: cases and analysis. Procedia - Social and Behavioral Sciences, vol. 54, pp. 598-607, 2012.

COZZOLINO, A.; ROSSI, S.; CONFORTI, A. Agile and lean principles in the humanitarian supply chain: the case of the United Nations world food programme. Journal of Humanitarian Logistics and Supply Chain Management, vol. 2, n. 1, pp. 16-33, 2012.

DAY, J. M.; MELNYK, S. A.; LARSON, P. D.; DAVIS, E. W.; WHYBARK, D. C. Humanitarian and disaster relief supply chains: a matter of life and death. Journal of Supply Chain Management, vol. 48, n. 2, pp. 21-36, 2012. 
ERTEM, M. A.; BUYURGAN, N.; ROSSETTI, M. D. Multiple-buyer procurement auctions framework for humanitarian supply chain management. International Journal of Physical Distribution \& Logistics Management, vol. 40, n. 3, pp. 202-227, 2010.

HAAVISTO, I.; KOVÁCS, G. Perspectives on sustainability in humanitarian supply chains. Disaster Prevention and Management, vol. 23, n. 5, pp. 610-631, 2014.

HERLIN, H.; PAZIRANDEH, A. Nonprofit organizations shaping the market of supplies. International Journal of Production Economics, vol. 139, n. 2, pp. 411-421, 2012.

IAKOVOU, E.; VLACHOS, D.; KERAMYDAS, C.; PARTSCH, D. Dual sourcing for mitigating humanitarian supply chain disruptions. Journal of Humanitarian Logistics and Supply Chain Management, vol. 4, n. 2, pp. 245-264, 2014.

KABRA, G.; RAMESH, A. Analyzing drivers and barriers of coordination in humanitarian supply chain management under fuzzy environment. Benchmarking: An International Journal, vol. 22, n. 4, pp. 559-587, 2015.

KOPCZAK, L.; JOHNSON, M. E. Rebuilding confidence: trust, control and information technology in humanitarian supply chains. In Academy of Management Annual Meeting, Philadelphia, Pennsylvania, United States, 2007.

KOVÁCS, G.; TATHAM, P. Humanitarian logistics performance in the light of gender. International Journal of Productivity and Performance Management, vol. 58, n. 2, pp. 174-187, 2009.

KOVÁCS, G.; SPENS, K. Identifying challenges in humanitarian logistics. International Journal of Physical Distribution \& Logistics Management, vol. 39, n. 6, pp. 506-528, 2009.

KOVÁCS, G.; SPENS, K. M. Humanitarian logistics and supply chain management: the start of a new journal. Journal of Humanitarian Logistics and Supply Chain Management, vol. 1, n. 1, pp. 5-14, 2011.

LEIRAS, A.; DE BRITO JR, I.; QUEIROZ PERES, E.; REJANE BERTAZZO, T.; TSUGUNOBU YOSHIDA YOSHIZAKI, $\mathrm{H}$. Literature review of humanitarian logistics research: trends and challenges. Journal of Humanitarian Logistics and Supply Chain Management, vol. 4, n. 1, pp. 95-130, 2014.

MAGHSOUDI, A.; PAZIRANDEH, A. Visibility, resource sharing and performance in supply chain relationships: insights from humanitarian practitioners. Supply Chain Management: An International Journal, vol. 21, n. 1, pp. 125-139, 2016.

MAON, F.; LINDGREEN, A.; VANHAMME, J. Developing supply chains in disaster relief operations through cross-sector socially oriented collaborations: a theoretical model. Supply Chain Management: An International Journal, vol. 14, n. 2, pp. 149-164, 2009.

MARTINEZ, A. J. P.; STAPLETON, O.; VAN WASSENHOVE, L. N. Field vehicle fleet management in humanitarian operations: a case-based approach. Journal of Operations Management, vol. 29, n. 5, pp. 404-421, 2011.

MAYS, R. E.; RACADIO, R.; GUGERTY, M. K. Competing constraints: the operational mismatch between business logistics and humanitarian effectiveness. In Global Humanitarian Technology Conference, 2012.

MCLACHLIN, R.; LARSON, P. D. Building humanitarian supply chain relationships: lessons 
from leading practitioners. Journal of Humanitarian Logistics and Supply Chain Management, vol. 1, n. 1, pp. 32-49, 2011.

MENEGHETTI, F. K. O que é um Ensaio-Teórico? Revista de Administração Contemporânea, vol. 15, n. 2, pp. 320-332, 2011.

OLORUNTOBA, R.; GRAY, R. Humanitarian aid: an agile supply chain? Supply Chain Management: An International Journal, vol. 11, n. 2, pp.115-120, 2006.

OLORUNTOBA, R. An analysis of the Cyclone Larry emergency relief chain: Some key success factors. International Journal of Production Economics, vol. 126, n. 1, pp. 85-101, 2010.

OVERSTREET, R. E.; HALL, D.; HANNA, J. B.; KELLY RAINER JR, R. Research in humanitarian logistics. Journal of Humanitarian Logistics and Supply Chain Management, vol. 1, n. 2, pp. 114-131, 2011.

PAZIRANDEH, A. Sourcing in global health supply chains for developing countries: Literature review and a decision making framework. International Journal of Physical Distribution \& Logistics Management, vol. 41, n. 4, pp. 364-384, 2011.

PAZIRANDEH, A.; HERLIN, H. Unfruitful cooperative purchasing: a case of humanitarian purchasing power. Journal of Humanitarian Logistics and Supply Chain Management, vol. 4, n. 1, pp. 24-42, 2014.

PELLEGRIN-ROMEGGIO, F.; VEGA, D. Combination as the Key Competence for the Dynamic Assembly of Temporary Networks: The Role of the Pivot-Assembler. In: A Focused Issue on Building New Competences in Dynamic Environments. Emerald Group Publishing Limited, vol. 7, pp. 61-83, 2014.

PFEFFER, J. S.; SALANCIK, G. The external control of organizations: a resource dependence perspective. New York, 1978.

RENNEMO, S. J.; RØ, K. F.; HVATTUM, L. M.; TIRADO, G. A three-stage stochastic facility routing model for disaster response planning. Transportation Research Part E: Logistics and Transportation Review, vol. 62, pp. 116-135, 2014.

ROTTKEMPER, B.; FISCHER, K.; BLECKEN, A. A transshipment model for distribution and inventory relocation under uncertainty in humanitarian operations. Socio-Economic Planning Sciences, vol. 46, n. 1, pp. 98-109, 2012.

SCHULZ, S. F.; BLECKEN, A. Horizontal cooperation in disaster relief logistics: benefits and impediments. International Journal of Physical Distribution \& Logistics Management, vol. 40, n. 8/9, pp. 636-656, 2010.

SEYBOLT, T. B. Harmonizing the humanitarian aid network: Adaptive change in a complex system. International Studies Quarterly, vol. 53, n. 4, pp. 1027-1050, 2009.

STEPHENSON Jr, M. Making humanitarian relief networks more effective: operational coordination, trust and sense making. Disasters, vol. 29, n. 4, pp. 337-350, 2005.

STEPHENSON JR, M.; SCHNITZER, M. H. Interorganizational trust, boundary spanning, and humanitarian relief coordination. Nonprofit Management and Leadership, vol. 17, n. 2, pp. 211-233, 2006. 
VAN DER LAAN, E. A.; DE BRITO, M. P.; VERGUNST, D. A. Performance measurement in humanitarian supply chains. International Journal of Risk Assessment and Management, vol. 13, n. 1, pp. 22-45, 2009.

VAN WASSENHOVE, L. N. Humanitarian aid logistics: supply chain management in high gear. Journal of the Operational Research Society, vol. 57, n. 5, pp. 475-489, 2006.

VEGA, D.; ROUSSAT, C. Humanitarian logistics: the role of logistics service providers. International Journal of Physical Distribution \& Logistics Management, vol. 45, n. 4, pp. 352-375, 2015.

YU, D.; YALCIN, M. G.; OZPOLAT, K.; HALES, D. N. Research in Humanitarian Supply Chain Management and a New Framework. Eurasian Journal of Business and Economics, vol. 8, n. 15, pp. 39-60, 2015.

Recebido em: 10-9-2017

Aprovado em: 1-6-2019

Avaliado pelo sistema double blind review.

Editor: Coordenação do PPGA/UMESP

Disponível em http://mjs.metodista.br/index.php/roc 